\title{
Erratum
}

\section{Erratum to: On the Ginzburg-Landau Functional in the Surface Superconductivity Regime}

\author{
M. Correggi ${ }^{1}$, N. Rougerie ${ }^{2}$ \\ ${ }^{1}$ Dipartimento di Matematica, "Sapienza" Università di Roma, P.le Aldo Moro 5, 00185 Rome, Italy. \\ E-mail: michele.correggi@gmail.com \\ 2 Université de Grenoble 1 and CNRS, LPMMC, Maison des Magistères CNRS, BP166, \\ 38042 Grenoble Cedex, France
}

Received: 23 December 2014 / Accepted: 26 February 2015

Published online: 8 May 2015 - @ S Springer-Verlag Berlin Heidelberg 2015

Commun. Math. Phys. 332, 1297-1343 (2014)

Through the refereeing process of a follow-up paper [1], we became aware of a mistake that affects the statement and proof of one of the main results (Theorem 2.4) formulated in the original article. In addition, some heuristic discussion has to be modified accordingly, but the other results are unaffected.

The mistake originated from the change of gauge described in Proposition 4.1, which is associated with the extraction of some suitable phase $\phi_{\varepsilon}(s, t)$ from the GinzburgLandau order parameter. Such a phase factor was unintentionally dropped in the rest of the discussion, generating the mistake mentioned above. To give an explicit example, the heuristic behavior of the order parameter in (1.16) must be replaced with

$$
\Psi^{\mathrm{GL}}(\mathbf{r}) \approx f_{0}\left(\frac{\eta}{\varepsilon}\right) \exp \left(-i \alpha_{0} \frac{\xi}{\varepsilon}\right) \exp \left(i \phi_{\varepsilon}\left(\frac{\xi}{\varepsilon}, \frac{\eta}{\varepsilon}\right)\right)
$$

We omit the extensive list of such corrections and refer to the revised version of the original paper [2]. For the sake of clarity we present, however, the correct statement of Theorem 2.4:

Theorem 2.4 (Winding number of $\Psi^{\mathrm{GL}}$ on the boundary of disc samples). Let $\Omega$ be $a$ disc of radius $R=k^{-1}$. For any $1<b<\Theta_{0}^{-1}$ as $\varepsilon \rightarrow 0$, any GL minimizer $\Psi^{\mathrm{GL}}$ satisfies

$$
\operatorname{deg}\left(\Psi^{\mathrm{GL}}, \partial \Omega\right)=\frac{\pi R^{2}}{\varepsilon^{2}}+\frac{\left|\alpha_{k}\right|}{\varepsilon}+\mathcal{O}\left(\varepsilon^{-3 / 4}|\log \varepsilon|^{2}\right) .
$$




\section{References}

1. Correggi, M., Rougerie, N.: Boundary behavior of the Ginzburg-Landau order parameter in the surface superconductivity regime. Arch. Rational Mech. Anal. (2014, to appear). arXiv:1406.2259 [math-ph]

2. Correggi, M., Rougerie, N.: On the Ginzburg-Landau functional in the surface superconductivity regime. (2014). arXiv:1309.2268 [math-ph] 